Accelerator Development Department Accelerator Physics Division BROOKHAVEN NATIONAL LABORATORY Associated Universities, Inc. Upton, NY 11973

Accelerator Physics Technical Note No. 20

Tracking Studies on a Lattice for the

SSC Low Energy Booster

\author{
G.F. Dell
}

April 1990 


\title{
Tracking Studies on a Lattice for the \\ SSC Low Energy Booster
}

\author{
G.F. Dell \\ Accelerator Development Department \\ April 9, 1990
}

\begin{abstract}
The aperture of the proposed lattice for the SSC Low Energy Booster (LEB) has been determined by particle tracking. Parallel edge dipoles are used, chromaticity is corrected with two families of sextupoles, and all magnets are assumed to be perfect (no multipoles). Tracking studies indicate a strong dependence of tune on emittance as well as a rather small acceptance ( $24 \mathrm{~mm}$ with a $40 \mathrm{~mm}$ vacuum chamber) when the chromaticity is corrected to zero. Use of partial chromaticity correction reduces the tune dependence on emittance and increases the acceptance to a value consistent with the size of the vacuum chamber.
\end{abstract}

\section{Lattice}

The lattice ${ }^{1}$ has a periodicity of six; each superperiod contains nine cells. The dispersion at the center of each superperiod is reduced to near zero values by removing the dipole from one half cell in cells 4 and 6 and both dipoles from cell 5. Chromaticity is corrected with two families of sextupoles (SF and SD) placed downstream of the QF and QD quadrupoles. The small negative dispersion at the center of each superperiod requires larger strength sextupoles if they are located in all half cells. Hence, two SF and one SD sextupoles have been removed from the center of each superperiod; this results in lower sextupole strength and increased dynamic aperture. Some lattice parameters are listed in Table 1.

1 LEB Lattice. Included in Superconducting Supercollider Site Specific Conceptual Design (Draft Jan. 1990). 


\section{Table 1. Typical Parameters of the SSC LEB Lattice at}

$$
\nu_{x}=16.850, \nu_{y}=16.750 \text {. }
$$

\begin{tabular}{llc} 
& QF & QD \\
\hline$\beta_{x}(\mathrm{~m})$ & 18.98 & 1.88 \\
$\beta_{y}(\mathrm{~m})$ & 1.90 & 19.22 \\
$\eta(\mathrm{m})$ & 1.06 & 0.22 \\
\hline & $\zeta_{x}^{N}$ & $\zeta_{y}^{N}$ \\
\hline Natural Chromaticity & -25.26 & -25.46 \\
\hline & $\mathrm{SF}$ & $\mathrm{SD}$ \\
\hline Sextupole Strength $\left(B^{\prime \prime} \ell / B \rho\right)$ & -0.7438 & 1.5022 \\
\hline
\end{tabular}

\section{Tracking}

Particle tracking has been performed with PATRICIA. ${ }^{2}$ Particles are launched with equal emittances in the $X$ and $Y$ planes. Two initial conditions are used: 1$)(X \neq 0$, $\left.Y \neq 0, X^{\prime}=Y^{\prime}=0\right)$, and 2$)\left(X \neq 0, Y^{\prime} \neq 0, X^{\prime}=Y=0\right)$. In the following text these will be referred to as Type 1 and Type 2 particles. An aperture test is made at the center and both ends of each dipole, at the center of each quadrupole, and at each sextupole to assure the particle remains within the specified aperture. Runs are repeated with larger and larger initial amplitudes until the test particle hits the aperture limit within 1000 turns. The principal tune of the particle motion is obtained by Fourier analysis.

The dependence of linear tune on momentum for full chromaticity correction is shown in Figure 1. The tunes are essentially unchanged over the momentum interval $-0.5 \leq$ $\Delta P / P \leq 0.5 \%$.

\section{Dynamic Aperture}

A test aperture of $1000 \mathrm{~mm}$ is used to determine the stability limit. At $\delta P / P=0$, this limit is reached for a Type 1 particle when the initial amplitudes, measured at the center of a horizontally focusing quadrupole, are $\left(X_{i}, Y_{i}\right)=(31 \mathrm{~mm}, 9.6 \mathrm{~mm})$. Dependence of the dynamic aperture on the degree of chromaticity correction is shown in Figure 2. Since the LEB will operate below transition energy, operation with partial or no chromaticity correction should be acceptable.

2 PATRICIA. H. Wiedemann, 1980 version. Multipole formulation added by S. Kheifets, SILAC. Further modifications as needed for use at BNL. 
A second working point, $\nu_{x}=16.850, \nu_{y}=10.750$, was also tried; its stability limit with full chromaticity correction is: $\left(X_{i}, Y_{i}\right)=(39.5 \mathrm{~mm}, 12.2 \mathrm{~mm})$. The findings are displayed on Figure 2.

\section{Physical Aperture}

The test $\sqrt{\left(X^{2}+Y^{2}\right)} \leq 40 \mathrm{~mm}$ is used in all elements. The results at the nominal working point are listed in Table 2 for the momentum interval $-0.5 \leq \Delta P / P \leq 0.5 \%$. We find Type 1 particles have a smaller acceptance than Type 2 particles.

\section{Table 2. Momentum Dependence of the $X$ Component of the Physical}

$$
\text { Aperture Measured at QF. } \epsilon_{x}=\epsilon_{y} . Y_{i}=0.31 X_{i} \text {. }
$$

Physical Aperture (mm) Tune: $\nu_{x}=16.850, \nu_{y}=16.750$.

$\begin{array}{llllcccc}\Delta P / P(\%) & -0.50 & -0.25 & -0.10 & 0.0 & 0.10 & 0.25 & 0.50 \\ (X, Y) & 23.8 & 23.8 & 23.8 & 23.8 & 23.8 & 23.8 & 23.8 \\ \left(X, Y^{\prime}\right) & 31.5 & 32.5 & 32.5 & 31.5 & 31.5 & 30.5 & 27.5\end{array}$

Plots of the dependence of $\nu_{x}$ and $\nu_{y}$ on emittance are shown in Figure 3 for both launching conditions. There is a strong dependence of tune on momentum that forces $\nu_{y}$ through the systematic resonance at $\nu_{y}=16.800$. Phase plots show five fold structure at this tune, but no instability could be produced.

Type 1 particles fail the $40 \mathrm{~mm}$ amplitude test when $x_{i}=24 \mathrm{~mm}$. At this amplitude both $\nu_{x}$ and $\nu_{y}$ (determined without the $40 \mathrm{~mm}$ restriction) exhibit a jump after which the dependence of tune of emittance is reduced. As the initial amplitude is increased, Type 1 particles again survive throughout 1000 turns for $25.6 \leq X_{i} \leq 26.7 \mathrm{~mm}$; all Type 1 particles having initial amplitudes $\geq 26.7 \mathrm{~mm}$ are lost within 1000 turns. See Figure 4.

The emittance function $\epsilon_{z}=\left(Z^{2}+\left(\alpha_{x} Z+\beta_{z} Z^{\prime}\right)^{2}\right) / \beta_{z}$ is determined at each revolution $(Z=X$ or $Y$ ). The amplitude of the motion is measured relative to a closed orbit whose coordinates are averages obtained from small amplitude motion. Smear plots are generated to show the relation between $\sqrt{\left(\epsilon_{y} / \epsilon_{y}[0]\right)}$ and $\sqrt{\left(\epsilon_{x} / \epsilon_{x}[0]\right)}$ turn by turn, where $\epsilon_{z}$ with $z=x, y$, or $t$ denotes $X, Y$, or total emittance, and $\left.\epsilon_{z}[0]\right)$ denotes the initial values of these quantities.

For linear, uncoupled motion, the smear plot should contain a single point at $\sqrt{\left(\epsilon_{y} / \epsilon_{y}(0)\right.}$ $=\sqrt{\left(\epsilon_{x} / \epsilon_{x}(0)\right)}=1$. Coupled motion for which $\epsilon_{x}+\epsilon_{y}=\epsilon_{t}(0)$ is represented by an arc having radius $\sqrt{2}$, and points with radial displacement $\neq \sqrt{2}$ indicate $\epsilon_{x}+\epsilon_{y} \neq \epsilon_{t}(0)$. 
Phase plots, smear plots, and plots of $\epsilon_{x}, \epsilon_{y}$ are shown in Figures 5 to 8 for a Type 1 and Type 2 particle having $\epsilon_{x} / \pi=\epsilon_{y} / \pi=20.77 \mathrm{~mm} \operatorname{mrad}\left(X_{i}=20 \mathrm{~mm}\right)$. The phase

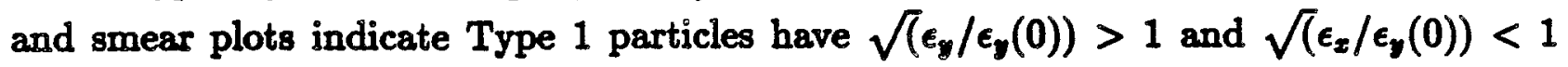
during much of the run. Figure 6(a) shows the increase in vertical and total emittance is immediate rather than being a result of gradual growth. Figure $6(\mathrm{~b})$ shows $\epsilon_{t}$ for 400 turns of a 1000 turn run; $\epsilon_{t}$ most often exceeds $\epsilon_{t}(0)$ throughout the run. Hence, Type 1 particles have large vertical amplitudes, and all test particles are lost in the vertically focusing quadrupoles.

In contrast, Type 2 particles have vertical and total emittance smaller than the initial values (Figure 8). Particles having initial amplitudes larger than $24 \mathrm{~mm}$ remain within the $40 \mathrm{~mm}$ test aperture; all Type 2 particles are lost in the horizontally focusing quadrupoles.

Plots of emittance $\epsilon_{x}, \epsilon_{y}$, and $\epsilon_{t}=\epsilon_{x}+\epsilon_{y}$ show that $\epsilon_{t} \geq \epsilon_{t}(0)$ for Type 1 particles and $\epsilon_{t} \leq \epsilon_{t}(0)$ for Type 2 particles. Tabulations of the physical aperture with full chromaticity correction $\zeta_{x}=\zeta_{y}=0$ and half chromaticity correction $\zeta_{x}=0.5 \zeta_{x}^{N}, \zeta_{y}=0.5 \zeta_{y}^{N}$ are listed in Table 3 for the normal operating point $\left(\nu_{x}, \nu_{y}\right)=(16.85,16.75)$ and the optional operating point $\left(\nu_{x}, \nu_{y}\right)=(16.85,10.75)$.

Table 3. $X$ Component of the Aperture for $100 \%$ and $50 \%$ Chromaticity Correction with $\epsilon_{x}=\epsilon_{y}$. Tune: $\nu_{x}=16.850, \nu_{y}=16.750$

\begin{tabular}{lllc} 
Aperture $X_{i}(\mathrm{~mm})$ & Dynam & Phys & Phys \\
Correction & $100 \%$ & $100 \%$ & $50 \%$ \\
$(X, Y)$ & 34.5 & 23.8 & 29.5 \\
$\left(X, Y^{\prime}\right)$ & 37.5 & 31.5 & 33.5 \\
& \multicolumn{2}{c}{ Tune: $\nu_{x}=16.850, \nu_{y}=10.750$} \\
Aperture $X_{i}(\mathrm{~mm})$ & Dynam & Phys & Phys \\
Correction & $100 \%$ & $100 \%$ & $50 \%$ \\
$(X, Y)$ & 39.5 & 33.5 & 36.5 \\
$\left(X, Y^{\prime}\right)$ & 40.5 & 34.5 & 37.5
\end{tabular}

$\underline{\text { Discussion }}$

A circular vacuum chamber having a $40 \mathrm{~mm}$ radius has been assumed. For $\epsilon_{x}=\epsilon_{y}$ one would expect; 1) an acceptance of $X_{i}=28.3 \mathrm{~mm}$ for complete coupling, and 2) an acceptance of $X_{i}=38.2 \mathrm{~mm}$ for zero coupling. Hence the acceptance of $23.8 \mathrm{~mm}$ obtained for the Type 1 particle (Tables 2 and 3 ) is unrealistically low and is attributed to the 
sextupoles for chromaticity correction. Acceptance values of $31.5 \mathrm{~mm}$ for the Type 2 particles could indicate lack of coupling, however the smear plots show the total emittance $\epsilon_{t}$ is less than $\epsilon_{t}(0)$, and the acceptance increases when chromaticity correction is changed from $100 \%$ to $50 \%$. Hence the acceptance of Type 2 particles is also influenced by the sextupoles.

In all cases, the particles are lost at locations of high $\beta$ even though $\Delta P / P$ is varied from $-0.5 \% \leq \Delta P / P \leq 0.5 \%$. As there is no observed loss in any dipoles, use of a flattened chamber with either axis less than $40 \mathrm{~mm}$ could be expected to reduce the acceptance of the LEB listed in Tables 2 and 3.

The strong tune dependence on emittance (Figure 3) should be expected to force the particles to cross important resonances. Reduction of the sextupole strength to that required for $50 \%$ chromaticity correction gives a significantly smaller dependence of tune shift on emittance (Figure 9). Operation with partial chromaticity correction to reduce the total tune spread (tune vs emittance plus tune dependence on momentum) should be considered. Fourier analysis of the particle motion indicates strong, principal peaks with little, if any indication of peaks near principal resonances, Figures 6(c) and 8(c).

Operation at the alternate working point $\nu_{x}=16.850, \nu_{y}=10.750$ results in lower chromaticity and in reduced coupling from sextupoles. Further improvement is obtained from partial chromaticity correction. Table 4 lists the natural chromaticities and sextupole strengths required for $100 \%$ and $50 \%$ chromaticity correction at $\nu_{x}=16.850, \nu_{y}=10.750$.

Table 4. Natural Chromaticity and Sextupole Strength Required for $100 \%$ and $50 \%$ Correction at $\nu_{x}=16.580, \nu_{y}=10.750$. $\zeta^{N}$ denotes Natural and $\zeta^{f}$ denotes Final Chromaticity

$100 \%$

$\mathrm{X}$

Y

$50 \%$

$\mathrm{X}$

$Y$

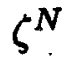

$-22.14$

$-15.62$

$$
\zeta^{N}
$$

$-22.14$

$-15.62$

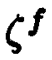

0.0

0.0

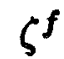

$-11.07$

$-7.81$
Strength

$$
-0.78250 \mathrm{~m}^{-1}
$$$$
1.20170 \mathrm{~m}^{-1}
$$

Strength

$-0.39130 \mathrm{~m}^{-1}$

$0.60091 \mathrm{~m}^{-1}$ 


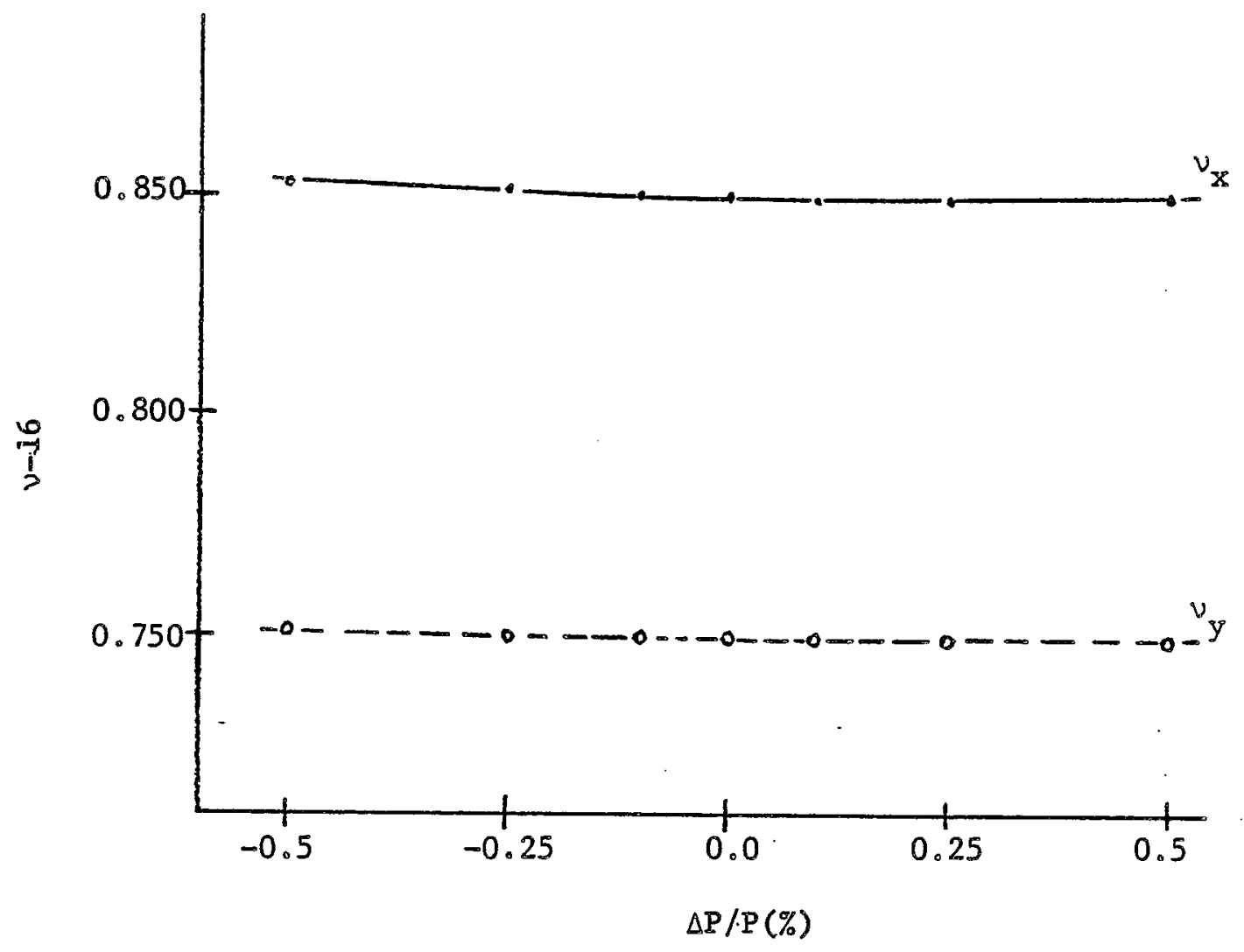

Figure 1. Dependence of the linear tune $\int d s / \beta$ on the momentum at the nominal operating point $\nu_{x}=16.850, \nu_{y}=16.750$. 


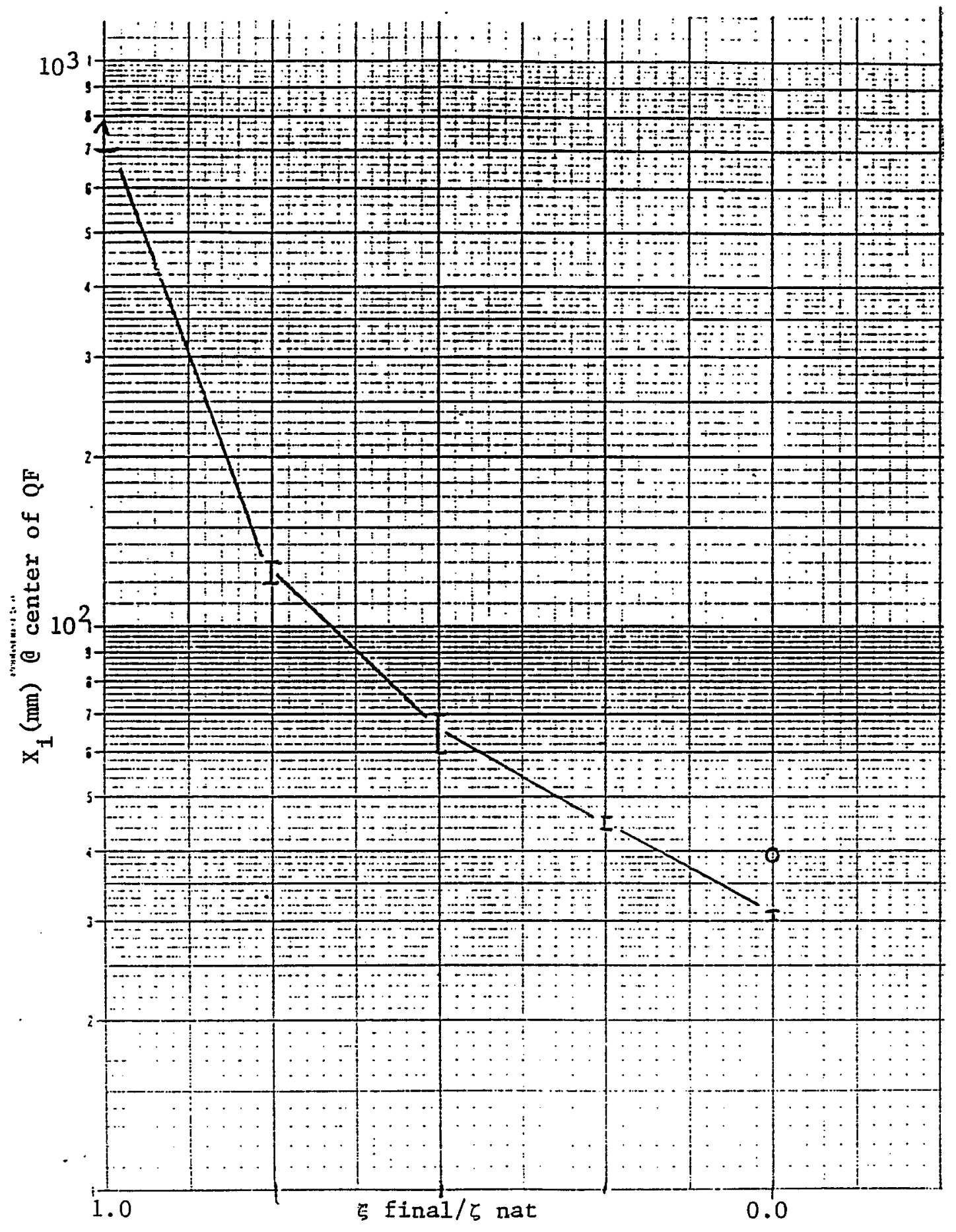

Figure 2. Dependence of the $\mathrm{X}$ component of the dynamic aperture on the degree of chromaticity correction. $\zeta^{f} / \zeta^{N}=0$ indicates the final chromaticity has been corrected to zero. Conditions: $\left.\epsilon_{x}=\epsilon_{y}, X_{i}=\sqrt{(} \beta_{x(Q F)} \epsilon_{x}\right)$ and $\left.Y_{i}=\sqrt{(} \beta_{y(Q F)} \epsilon_{y}\right)$.

I values for $\nu_{x}=16.85, \nu_{y}=16.75$.

$\mathrm{O}$ value for $\nu_{x}=16.85, \nu_{y}=10.75$. 

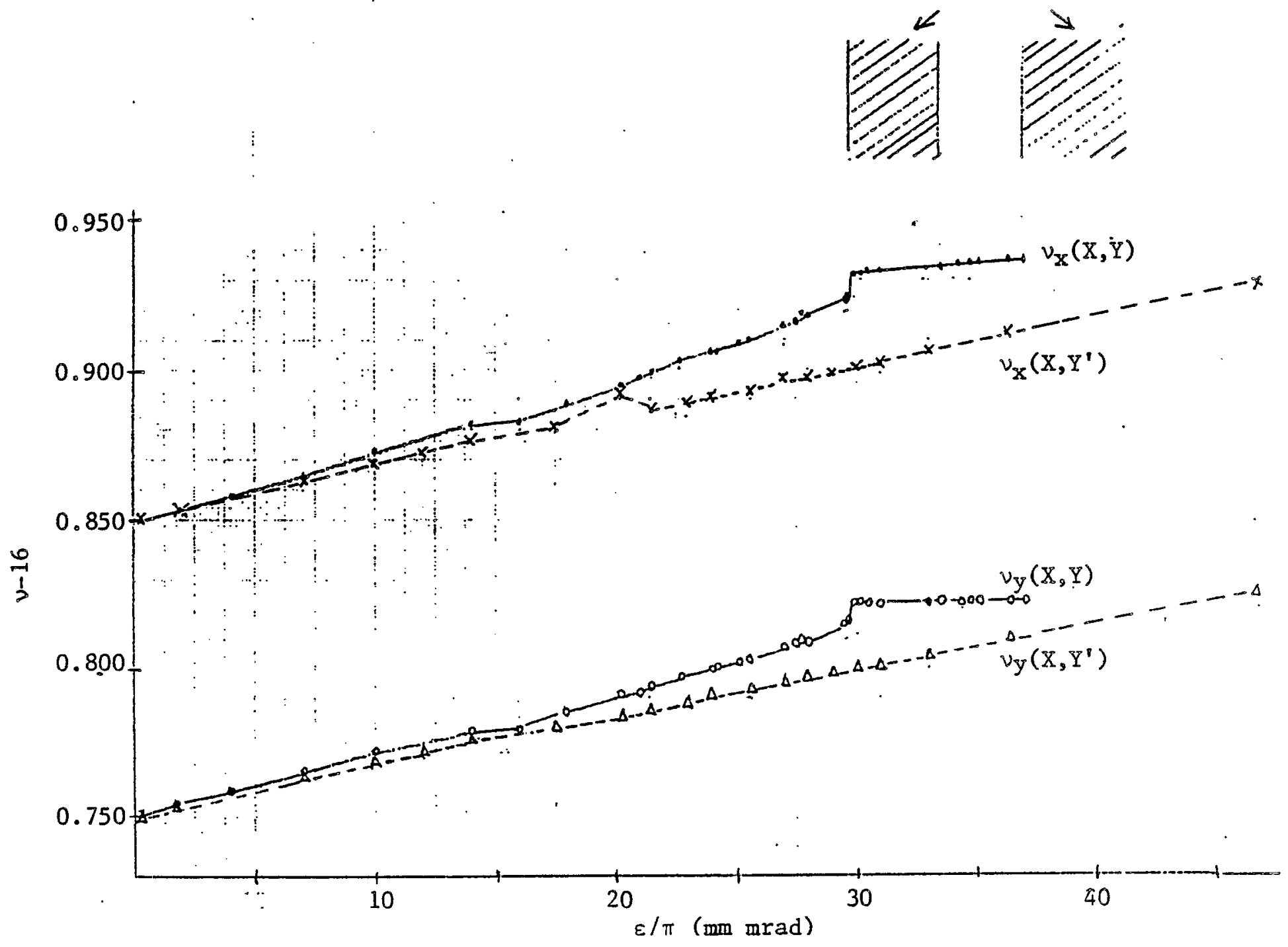

Figure 3. Dependence of tune on emittance at $\Delta P / P=0 \%$ for $100 \%$ chromaticity correction when $\epsilon_{x}=\epsilon_{y}=\epsilon_{0}$. Solid line indicates Type 1 particle with $X_{i} \neq 0, Y_{i} \neq 0, X_{i}^{\prime}=0$, and $Y_{i}^{\prime}=0$; dashed line indicates Type 2 particle with $X_{i} \neq 0, Y_{i}=0, X_{i}^{\prime}=0$, and $Y_{i}^{\prime} \neq 0$. 


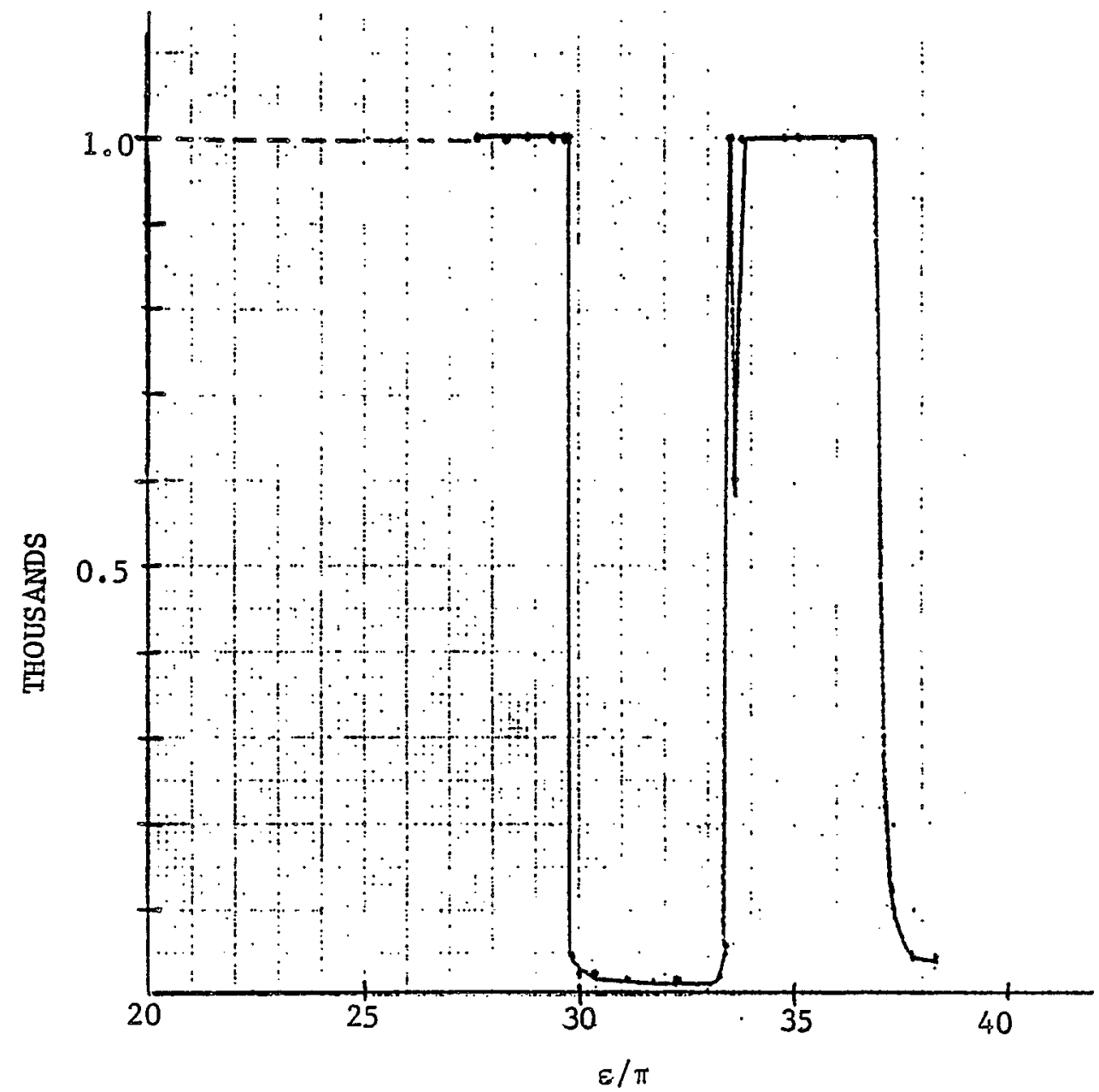

Figure 4. Detail of stability plot for Type 1 particle. The dip indicates a region where the amplitude of the particles has grown and exceeds the dimension of the vacuum chamber. The physical aperture at $\epsilon / \pi=30$ corresponds to $X_{i}=24 \mathrm{~mm}$. 

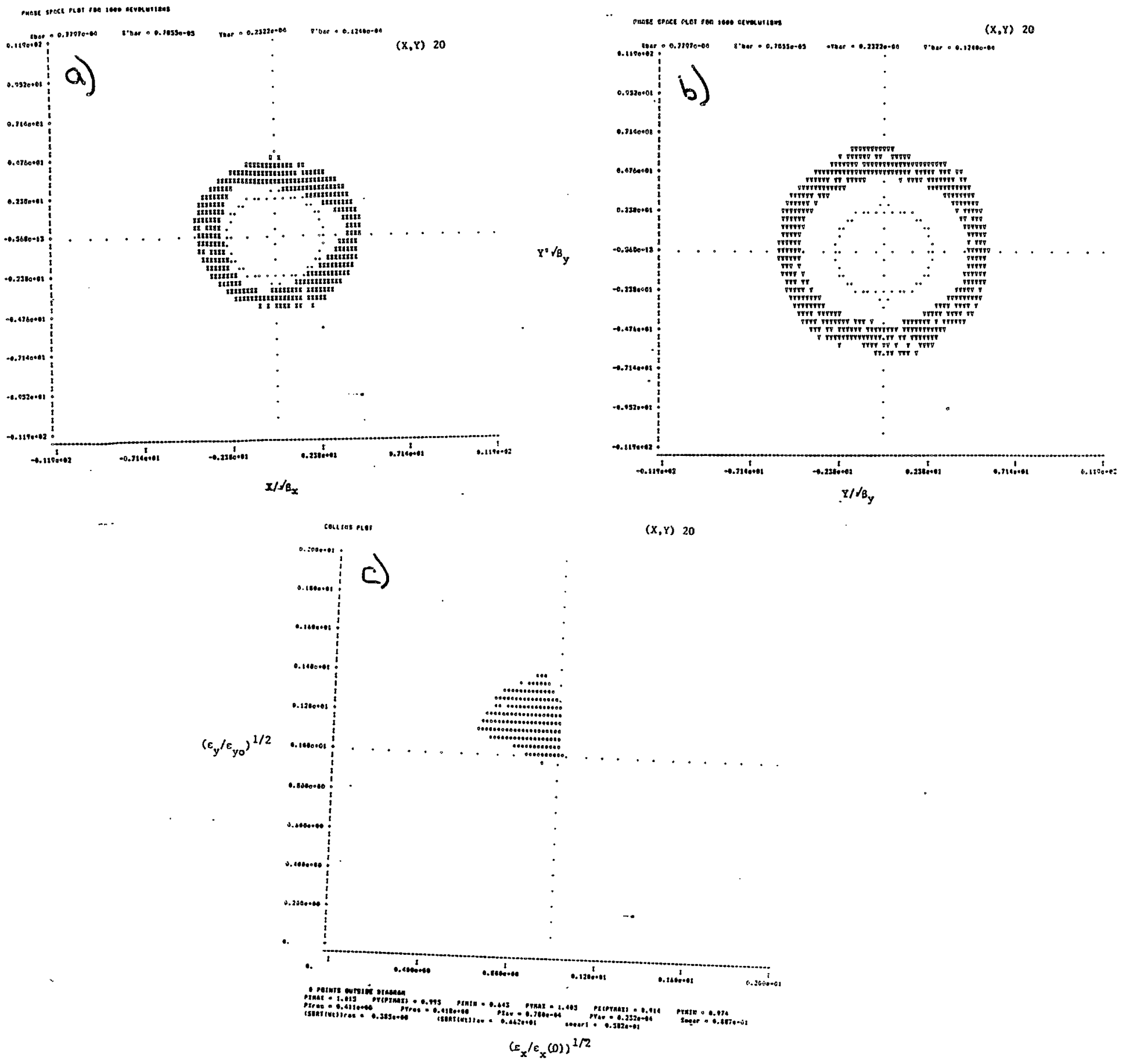

Figure 5. Phase and smear plots for Type 1 particle having $X_{i}{ }^{\prime}=20 \mathrm{~mm}, Y_{i}=6.3$ $\mathrm{mm} ; \epsilon_{x}(0)=\epsilon_{y}(0)$. a) Phase plot for horizontal motion (radius indicates $\sqrt{\epsilon}_{x}$ ), b) Phase plot for vertical motion, and c) smear plot showing the relation beteen $\left.\sqrt{(} \epsilon_{y} / \epsilon_{y}(0)\right)$ and $\left.\sqrt{(} \epsilon_{x} / \epsilon_{x}(0)\right)$ during the 1000 turn tracking run. The phase and smear plots indicate the $\mathrm{X}$ motion doesn't exceed its initial amplitude while the y motion usually exceeds its initial amplitude. Also, $\epsilon_{x}+\epsilon_{y} \geq \epsilon_{t}(0)$. 

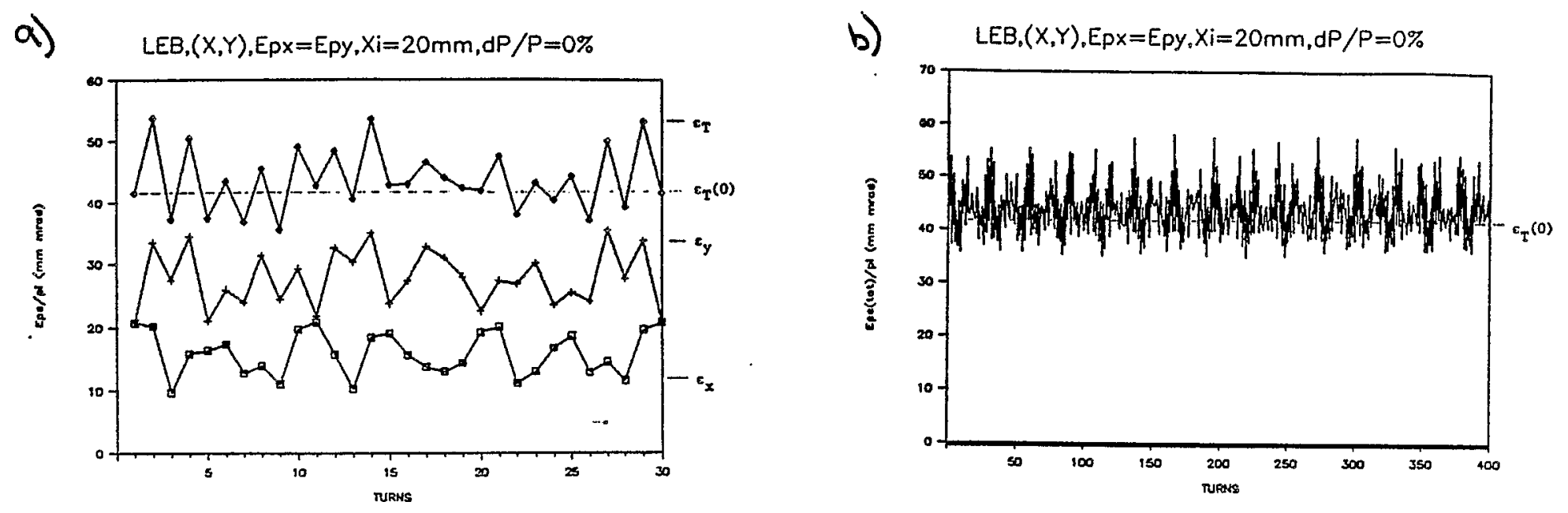

C)

LEB $,(X, Y), E p x=E p y, X i=20 \mathrm{~mm}, d P / P=0 \%$

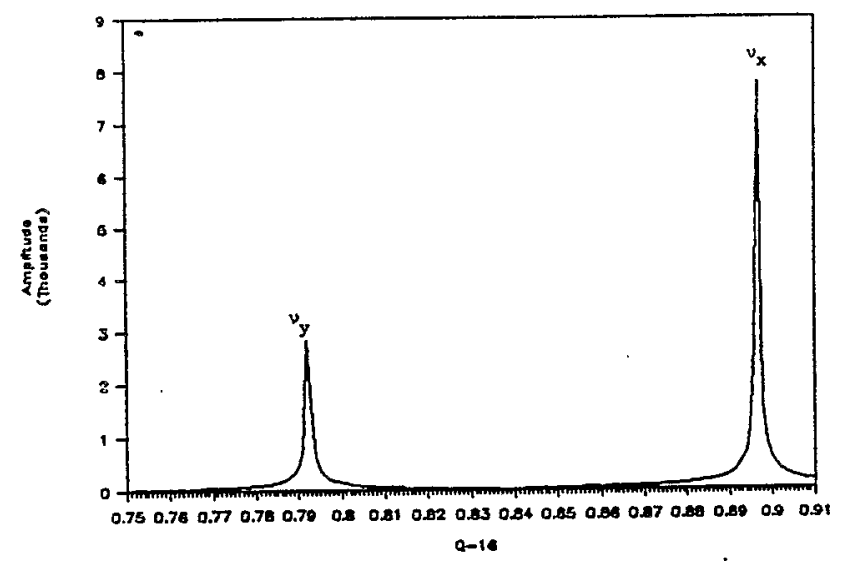

Figure 6. Plots for Type 1 particle showing: a) time dependence of $\epsilon_{x}, \epsilon_{y}$ and $\epsilon_{t}$ for the first 30 turns of a 1000 turn run, b) time dependence of $\epsilon_{t}=\epsilon_{x}+\epsilon_{y}$ during the first 400 turns of a 1000 turn run (large excursions of $\epsilon_{y}$ and $\epsilon_{t}$ are immedate - not the result of long term growth), and c) Fourier spectra showing $\nu_{x}$ and $\nu_{y}$ - only the principal peaks have significant amplitude. 
a)

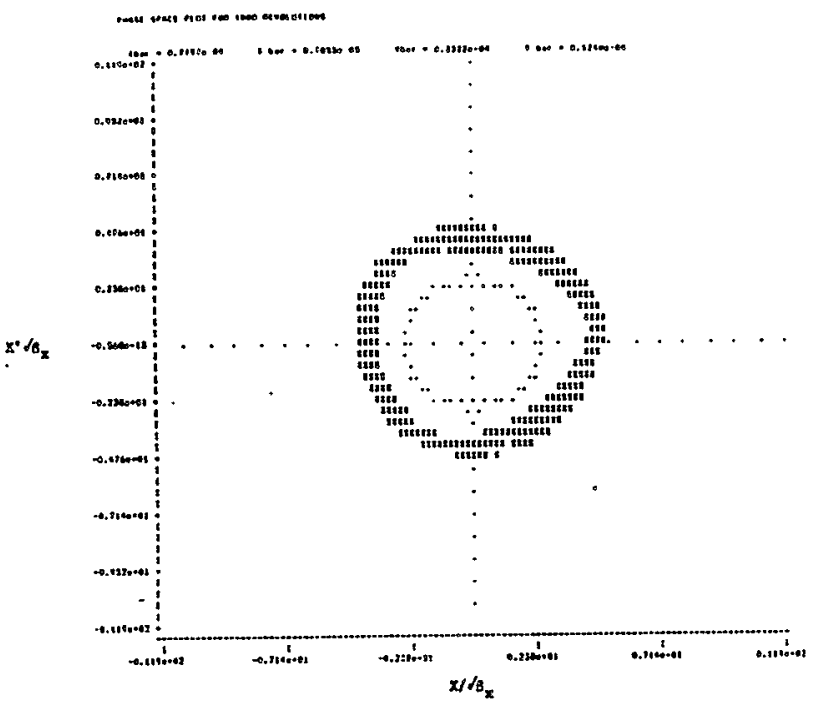

b)

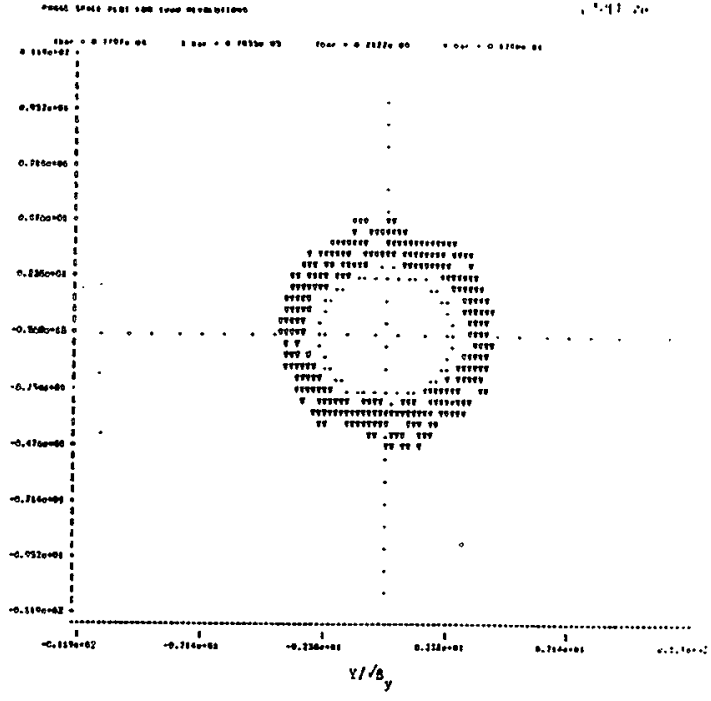

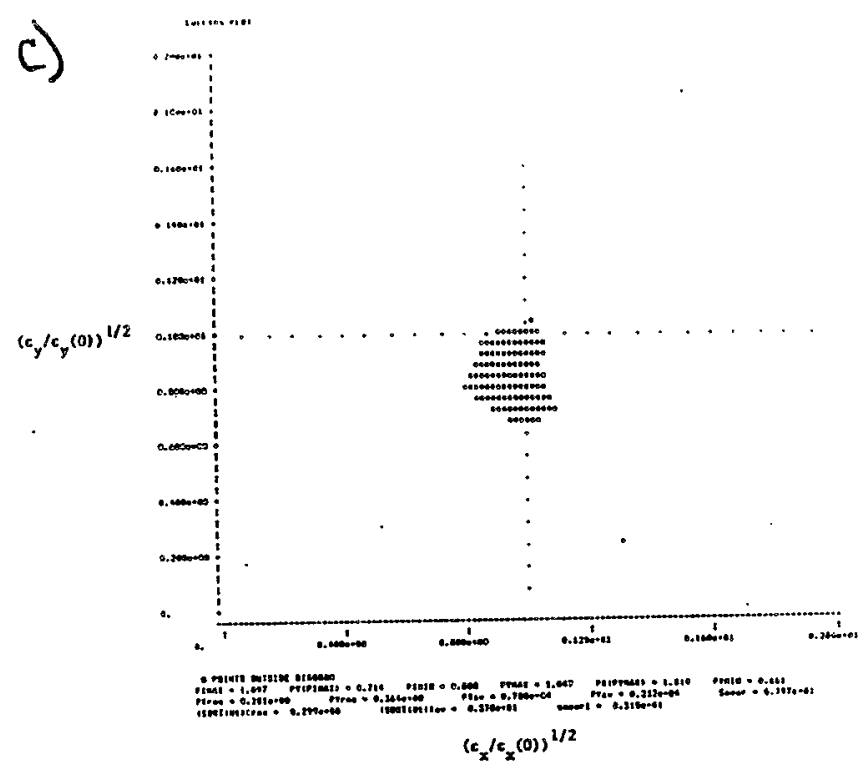

Figure 7. Phase and smear plots for Type 2 particles having $X_{i}=20 \mathrm{~mm}, Y_{i}^{\prime} \neq 0$; $\epsilon_{x}(0)=\epsilon_{y}(0)$. a) Phase plot for horizontal motion (radius indicates $\sqrt{\epsilon}_{x}$, b) Phase plot for vertical motion, and c) smear plot showing the relation between $\sqrt{\left(\epsilon_{y} / \epsilon_{y}(0)\right)}$ and $\left.\sqrt{(} \epsilon_{x} / \epsilon_{x}(0)\right)$ during the 1000 turn tracking run. The phase and smear plots indicate the $\mathrm{X}$ sometimes exceeds its initial amplitude while the $\mathrm{y}$ motion seldom exceeds its initial amplitude. Also, $\epsilon_{x}+\epsilon_{y} \leq \epsilon_{t}(0)$. 
a)
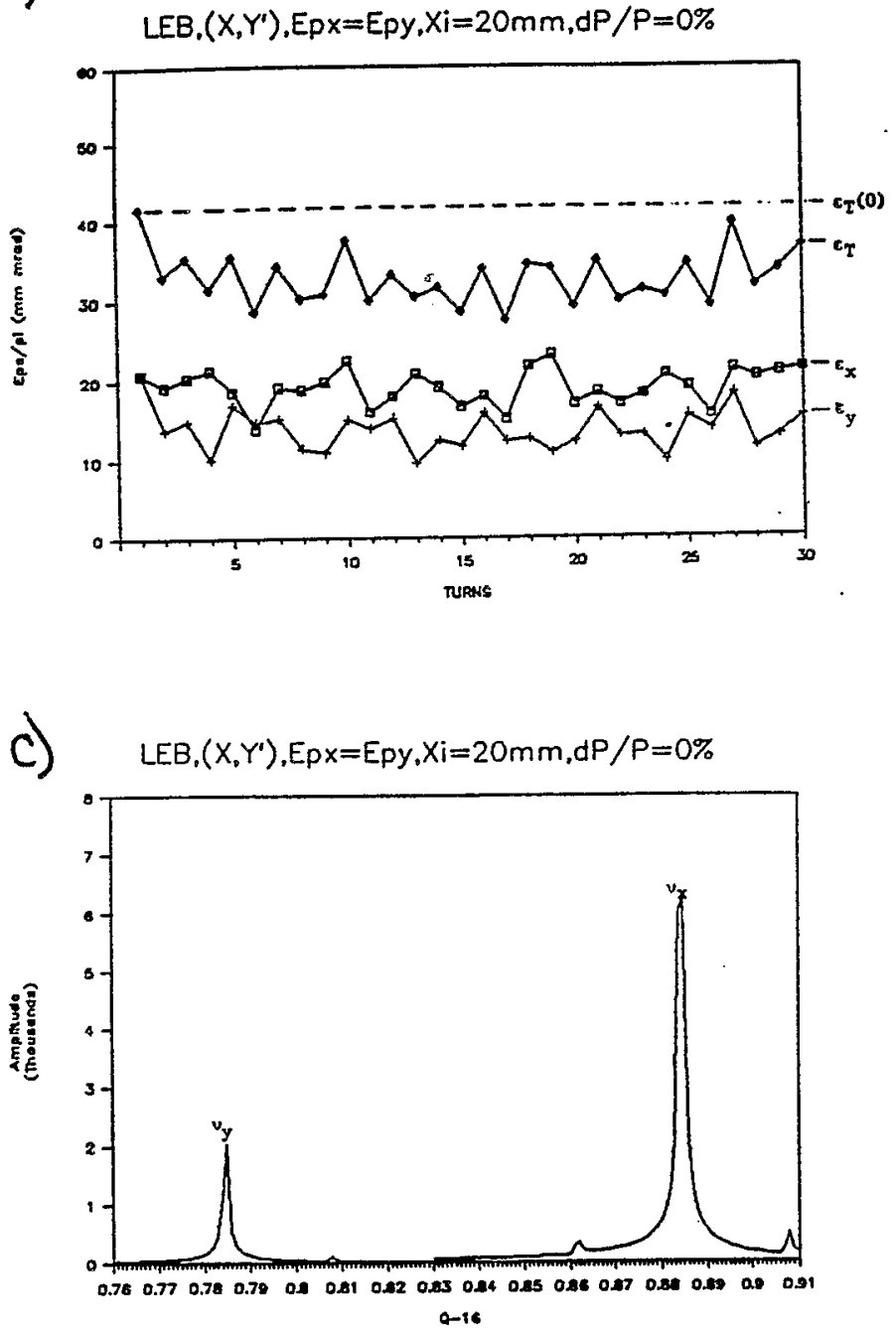

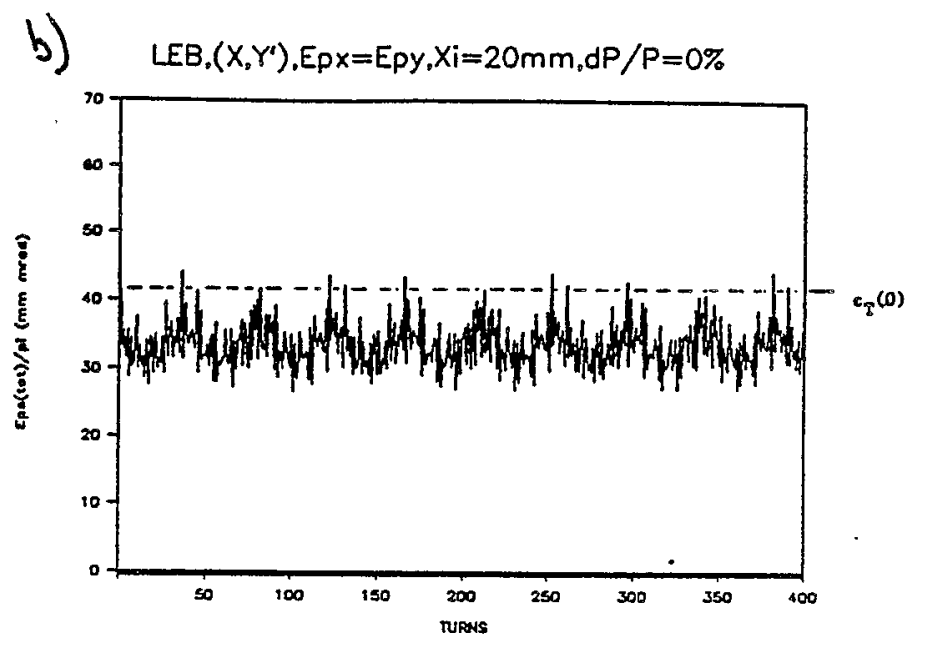

Figure 8. Plots for Type 2 particle showing: a) time dependence of $\epsilon_{x}, \epsilon_{y}$ and $\epsilon_{t}$ for the first 30 turns of a 1000 turn run, b) time dependence of $\epsilon_{t}=\epsilon_{x}+\epsilon_{y}$ during the first 400 turns of a 1000 turn run (total emittance $\epsilon_{t}$ is usually less than the initial value $\epsilon_{t}(0)$, and c) Fourier spectra showing $\nu_{x}$ and $\nu_{y}$. Small satellite peaks are present near the principal peaks. 


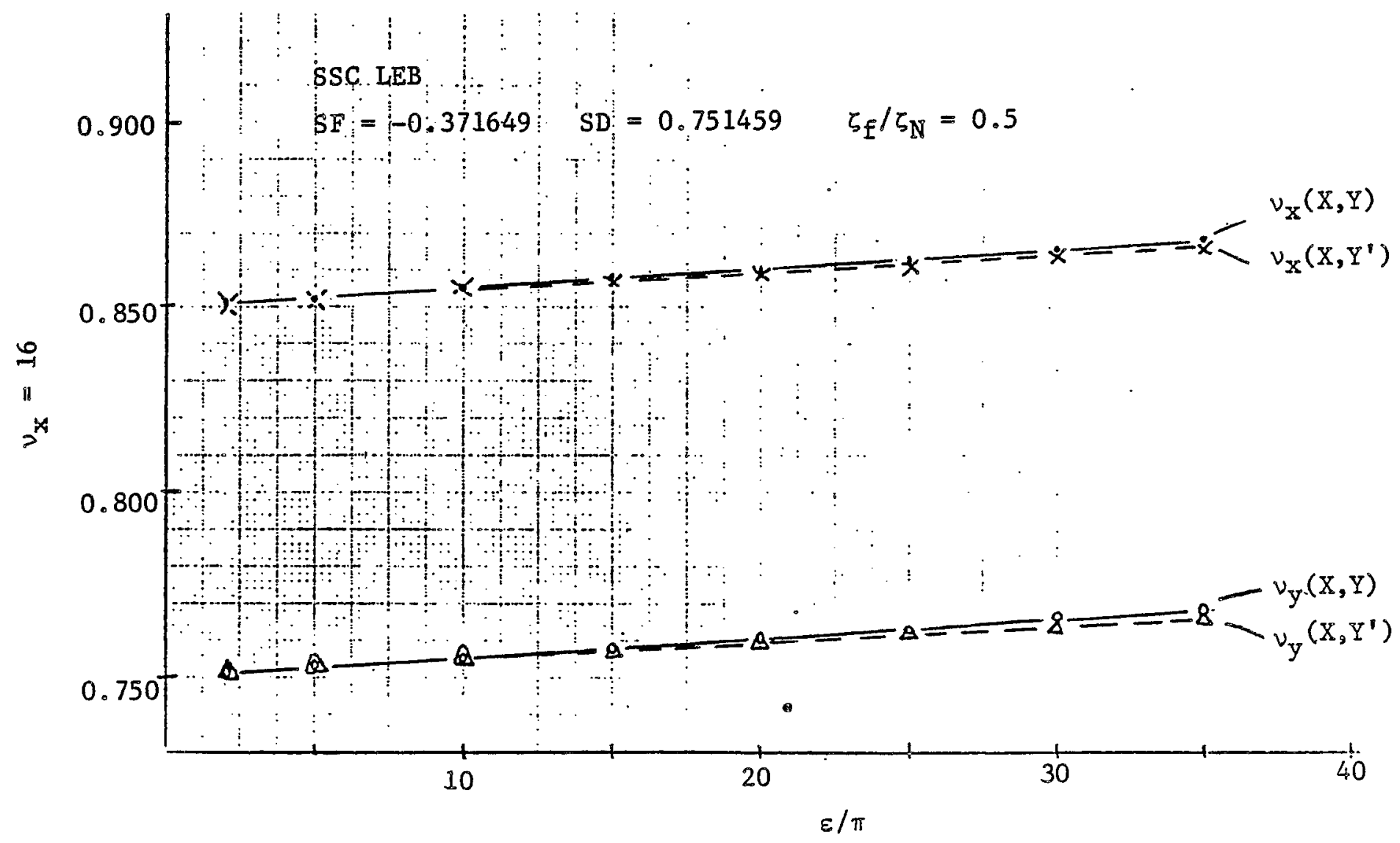

Figure 9. Dependence of tune on initial emittance $\epsilon(0)$ at $\Delta P / P=0 \%$ for $50 \%$ chromaticity correction when $\epsilon_{x}=\epsilon_{y}=\epsilon(0)$. Solid line indicates Type 1 particle baving $X_{i} \neq 0$, $Y_{i} \neq 0, X_{i}^{\prime}=0$, and $Y_{i}^{\prime}=0$; dashed line indicates Type 2 particle having $X_{i} \neq 0, Y_{i}=0$, $X_{\dot{i}}^{\prime}=0$, and $Y_{i}^{\prime} \neq 0$ 。 